\title{
Best Practices for real-time in situ Atomic Force Microscopy and Chemical Force Microscopy of Crystals
}

Laura N. Poloni, * Xiaodi Zhong*, Michael D. Ward, and Trinanjana Mandal*

*Ward Lab, Department of Chemistry and the Molecular Design Institute, New York University, 100 Washington Square East, New York, NY 10003-6688

\author{
Received Date: $\quad$; Email: tm1454@nyu.edu
}

\section{SUPPORTING INFORMATION}

Video S1. Still frame from instructional video of fluid cell assembly on Bruker Multimode 8 AFM.

Video S2. Still frame from instructional video of switching syringes during in situ AFM experiment on Bruker Multimode 8 AFM.

Video S3. Still frame from instructional video of fluid cell disassembly on Bruker Multimode 8 AFM.

Video S4. Still frame from instructional video of fluid cell assembly on Asylum MFP-3D-SA AFM.

Video S5. Still frame from instructional video of fluid cell mounting on and set up of Asylum MFP-3D-SA AFM. 


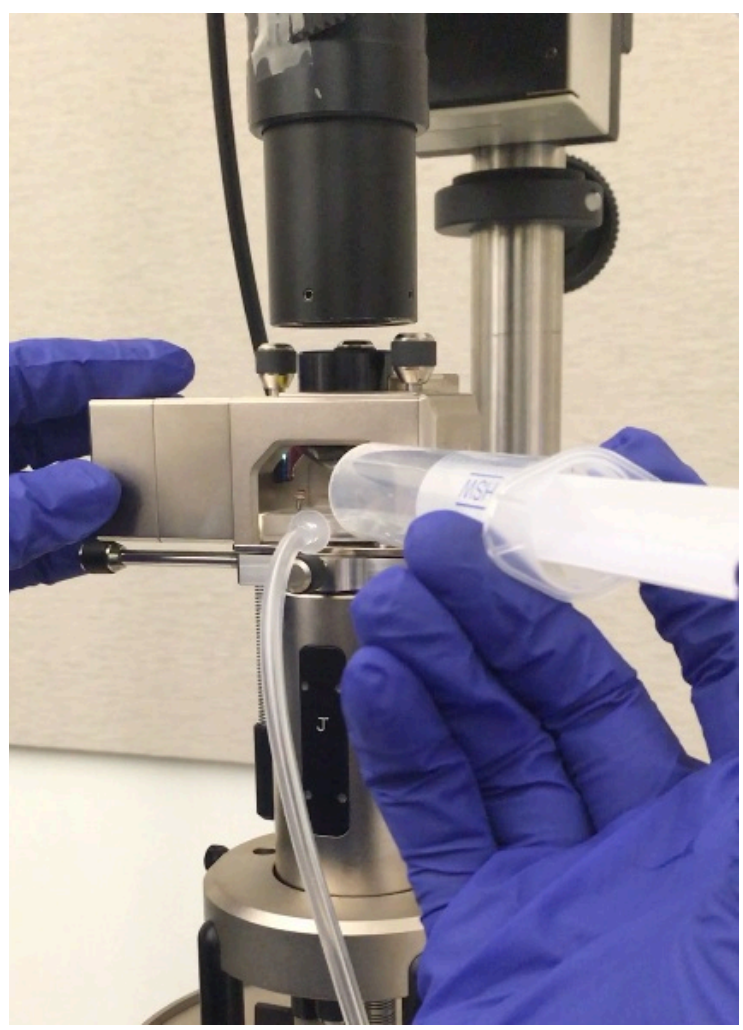

Video S1. Still frame from instructional video VideoS1_BrukerFluidCellSetup.MOV. Video depicts positioning of Oring on AFM fluid cell probe holder, mounting of fluid cell probe holder on AFM, connecting of outlet tubing, checking for leaks in the seal between O-ring and sample, and connecting of inlet tubing for in situ AFM experiments conducted on the Bruker Multimode 8 AFM.

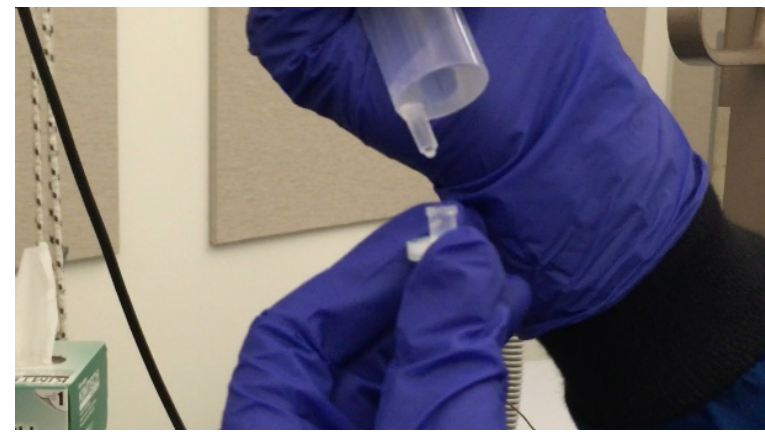

Video S2. Still frame from instructional video VideoS2_BrukerChangingSyringe.MOV. Video depicts changing syringe containing the crystal growth medium during in situ AFM experiments conducted on the Bruker Multimode 8 AFM. 


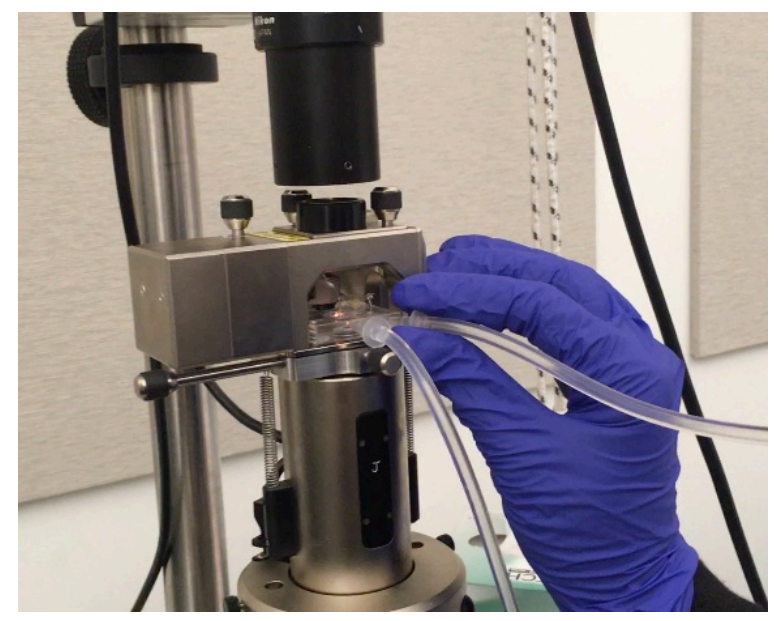

Video S3. Still frame from instructional video VideoS3_BrukerFluidCellDisassembly.MOV. Video depicts disassembly of experimental setup for in situ AFM experiments conducted on the Bruker Multimode 8 AFM.

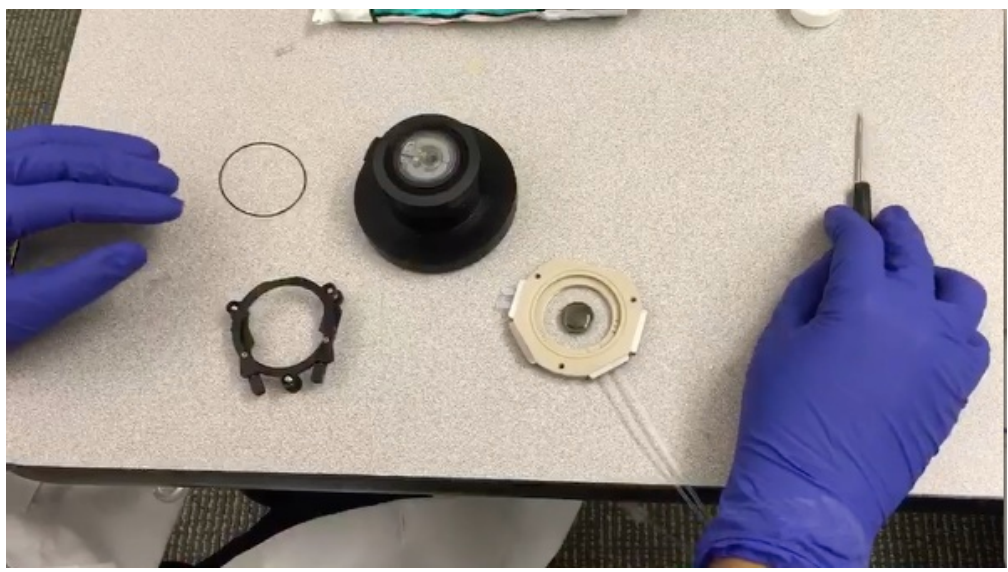

Video S4. Still frame from instructional video VideoS4_AsylumFluidCellAssembly.MOV. Video depicts assembly of closed fluid cell for in situ AFM experiments conducted on the Asylum MFP-3D-SA AFM.

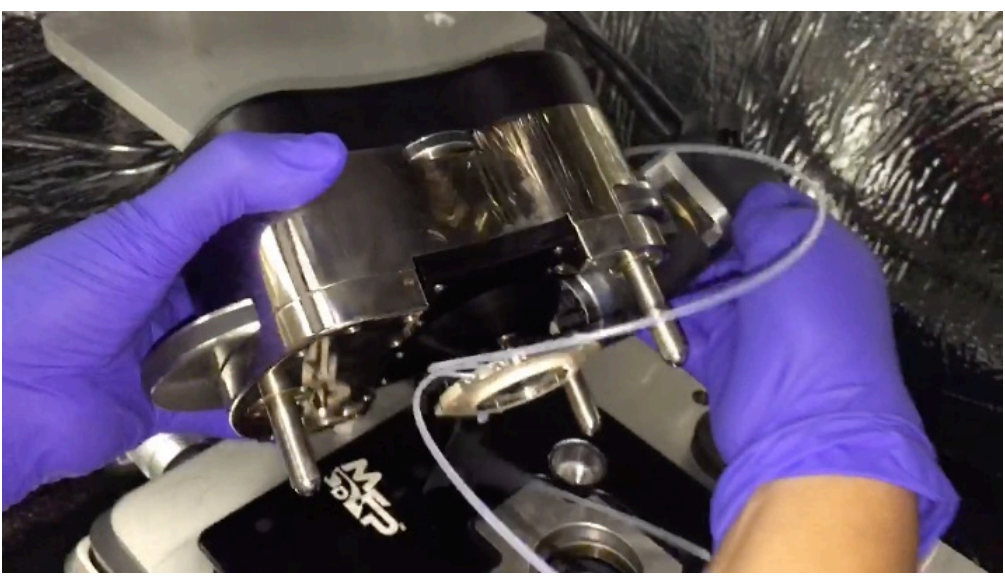

Video S5. Still frame from instructional video VideoS5_AsylumAssembly.MOV. Video depicts mounting of closed fluid cell on the AFM for in situ AFM experiments conducted on the Asylum MFP-3D-SA AFM. 